\title{
Dissection of the internal carotid artery and stroke after mandibular fractures: a case report and review of the literature
}

\author{
Ingrid Aune Tveita ${ }^{1 *} \mathbb{D}$, Martin Ragnar Skjerve Madsen² and Erik Waage Nielsen $3,4,5$
}

\begin{abstract}
Background: We present a report of a patient with blunt trauma and mandibular fractures who developed a significant cerebral infarction due to an initially unrecognized injury of her left internal carotid artery. We believe that increased knowledge of this association will facilitate early recognition and hence prevention of a devastating outcome.

Case presentation: A 41-year-old ethnic Norwegian woman presented to our Emergency Room after a bicycle accident that had caused a direct blow to her chin. At admittance, her Glasgow Coma Scale was 15. Initial trauma computed tomography showed triple fractures of her mandible, but no further pathology. She was placed in our Intensive Care Unit awaiting open reduction of her mandibular fractures. During the following 9 hours, she showed recurrent episodes of confusion and a progressive right-sided hemiparesis. Repeated cerebral computed tomography revealed no further pathology compared to the initial scan. She had magnetic resonance angiography 17 hours after admittance, which showed dissection and thrombus formation in her left internal carotid artery, total occlusion of her left medial cerebral artery, and left middle cerebral artery infarction was detected.
\end{abstract}

Conclusions: Carotid artery dissection is a rare but life-threatening condition that can develop after trauma to the head and neck. There should be a high index of suspicion in patients with a mechanism of injury that places the internal carotid artery at risk because blunt vascular injury may show delayed onset with no initial symptoms of vascular damage. By implementing an algorithm for early detection and treatment of these injuries, serious brain damage may be avoided.

Keywords: Blunt cerebrovascular injury, Mandibular fracture, Carotid artery dissection, Facial trauma, Blunt vascular injury

\section{Background}

The case presented illustrates the link between facial fractures and blunt cerebrovascular injury (BCI) that, falsely, has long been considered a curiosity. In order to identify these patients at an early stage this case report emphasizes the need for implementation of appropriate screening protocols in the Emergency Room (ER). This was not the case at our hospital at the time the actual patient was admitted, and hence diagnosis was delayed with consequences for patient outcome.

\footnotetext{
* Correspondence: ingrid.aune.tveita@nordlandssykehuset.no

${ }^{1}$ Department of Ear Nose and Throat Surgery, Nordland Hospital, Bodø,

Norway

Full list of author information is available at the end of the article
}

$\mathrm{BCI}$ has long been considered a curiosity, and may explain why clinically recognizable neurological symptoms often occur before diagnosis is made [1]. Early reports suggest mortality rates of $28 \%$, and subsequent multicenter reviews have confirmed these rates, with 48 to $58 \%$ of survivors having permanent severe neurological deficits [2].

The incidence of $\mathrm{BCI}$ among all patients experiencing blunt trauma in the United States of America (USA) is estimated at approximately $0.1 \%$, rising to $1.6 \%$ with initiation of screening [2-4].

Early antithrombotic intervention has the potential to improve neurologic outcome, given that $\mathrm{BCI}$ is confirmed and that no contraindications for this treatment exist (for example, bleeding pelvic fracture) [5-7]. 


\section{Case presentation}

A healthy, non-tobacco smoking, 41-year-old ethnic Norwegian woman presented to our ER 10 minutes after a bicycle accident. On arrival, she was conscious and complained of jaw pain. She explained that she, after rapid deceleration, had fallen over the handlebars and landed on her face. She was wearing a helmet. A trauma assessment was initiated: her Airway, Breathing, Circulation, Disability, Exposure assessment and Glasgow Coma Scale (GCS) were normal. A physical examination revealed blood pressure $130 / 60 \mathrm{mmHg}$, respiratory rate 20 breaths per minute, and normal auscultatory findings of her heart and lungs. Her blood tests showed normal complete blood count $(\mathrm{CBC})$, bleeding status, and coagulation status, as well as liver and renal function.

A trauma computed tomography (CT) scan reported normal brain status, but fractures of the mandible were found in both condylar necks and the left paramedian corpus (Fig. 1).

She was taken to our Intensive Care Unit (ICU) and, due to stable fractures, the surgery was planned for the next morning. She became confused and had several bouts of tachycardia 1.5 hours after admittance. She responded to verbal contact but was unable to follow instructions. After a few minutes she seemed more alert and took instructions more actively.

A quick neurological examination was made, with the only concern being a slightly impaired finger-nose test on the right side.

The evening passed with a few more episodes of brief confusion.

A repeated neurological examination gave suspicion of brain stem involvement with a decline in GCS to 7 (M 5, $\mathrm{V}$ 1, E 1). A repeated CT scan, 10 hours after admission,

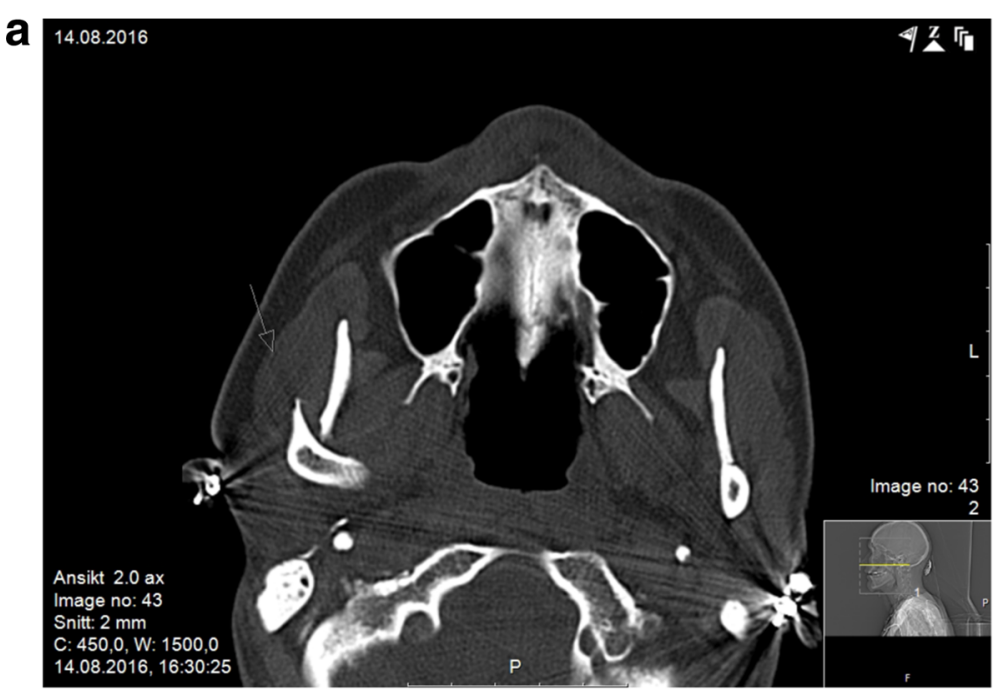

b

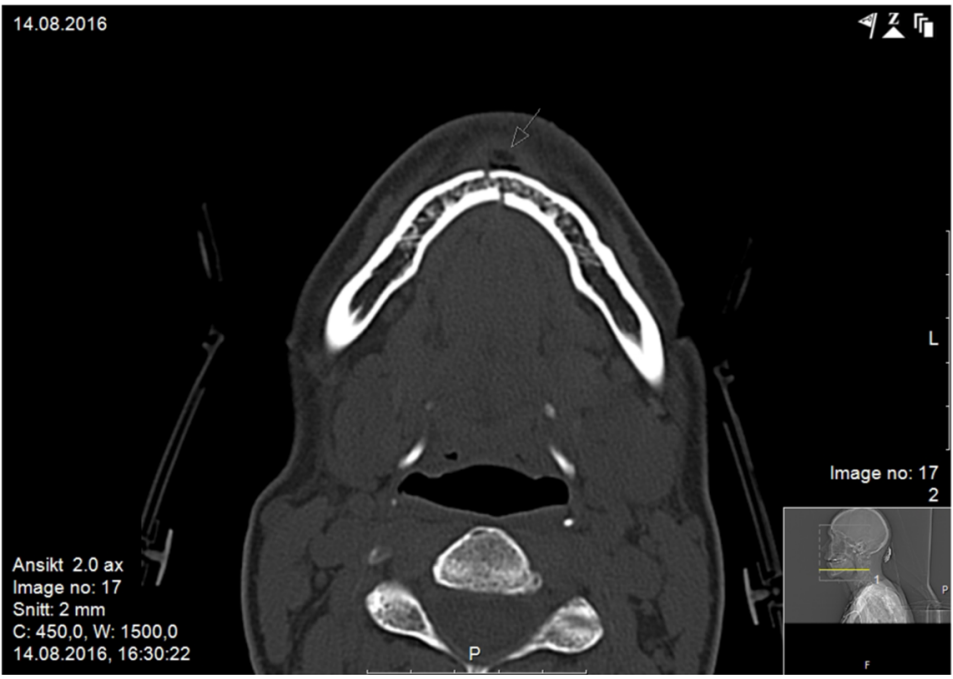

Fig. 1 a, b Computed tomography scan at admittance shows fractures of the mandible (bilateral condylar neck and left corpus paramedian) 
showed no recent changes. Unfortunately, no further immediate radiologic assessment was initiated.

Her vital parameters were stable; magnetic resonance imaging (MRI) was planned for the next morning.

The following morning she presented with palsy in her right extremities and acquired anisocoria with a larger right pupil. She had a GCS of 6 (M 4, V 1, E 1). She was intubated and an MRI and magnetic resonance angiography (MRA) of her head and neck were conducted 17 hours after admission. An extensive left middle cerebral artery infarction was detected, with cessation of diffusion in the supply area of her left middle cerebral artery (MCA). Further, an occluded left internal carotid artery (ICA) was diagnosed, approximately $1 \mathrm{~cm}$ above the bifurcation, in addition to occlusion of her left MCA (Fig. 2).

She was given $300 \mathrm{mg}$ acetylsalicylic acid intravenously to reduce risk for progressive thrombosis, and immediately transferred to a level 1 trauma center where she received neurointensive care and a pressure-releasing hemicraniectomy. Postoperatively, CT angiography and ultrasound of her carotid arteries were made and confirmed dissection of her left ICA with total occlusion of the vessel. In addition, dissection and a moderate-grade to high-grade stenosis of $40 \%$ of her right ICA was found.

She was treated with dual platelet inhibition, and fixation of her mandibular fractures was postponed. After 4. days, she was extubated and transferred to our neurological department for rehabilitation. The mandibular fractures were fixated 8 days after the initial trauma. She still has aphasia and a right-sided hemiplegia 2 months after the bicycle injury.

The mechanisms of vessel injury in this case are probably a combination of linear and torsional forces due to hyperextension. Direct pressure from her dislocated mandibular bone may have contributed. Bouts of tachycardia could stem from a disturbed carotid body or from cerebral ischemic insults.

\section{Discussion}

In the case presented, a healthy woman presented with clinical signs of rapid deterioration in neurological status 10 hours after admission to our hospital due to a blunt trauma to her chin. In retrospect, the repeat CT scan showing no recent changes was insufficient to rule out cerebrovascular damage, and should have prompted follow-up angiographic imaging. Unfortunately, this was delayed and 17 hours after admission cerebral MRI and MRA detected an extensive left middle cerebral artery infarction caused by a thrombus occluding her left MCA.

Due to the late diagnosis of a $\mathrm{BCI}$, antithrombotic intervention was delayed until 20 hours after admittance.

It is not known whether earlier diagnostic screening and intervention would have altered our patient's outcome, but the literature covering $\mathrm{BCI}$ indicates that early recognition and treatment of this condition have the potential to reduce fatal outcome. Antithrombotic therapy is usually the only treatment option as surgical repair is not possible due to the location of the thrombus. Thus, early antithrombotic treatment of our patient might have halted progression of thrombosis, and thus possibly reduced the chance of development of serious focal neurological deficit.

Searching for case reports on the subject, there are few records of cases with patients presenting clear symptoms of cerebrovascular injury at hospital admittance. Insight of the mechanisms of trauma associated with $\mathrm{BCI}$ is thus crucial for all health care providers working with trauma care. This knowledge will raise awareness and ensure recognition of patients at risk for $\mathrm{BCI}$, and prompt early and appropriate diagnostic screening allowing early institution of treatment.

To facilitate our awareness and recognition of this condition, we present a synopsis of a literature search on BCI emphasizing pathophysiology, screening options, and treatment.

\section{Pathophysiology}

High impact trauma to the neck or face is a potential risk for BCI. An increased awareness of this relationship is crucial to facilitate early recognition of the injury and to initiate early intervention to prevent further complications [8].

Four characteristic mechanisms of blunt carotidal injury are listed in Table 1 [9].

The pathophysiological cascade is believed to be an intimal tear of the artery created by a force that twists or stretches the vessel, or the vessel is impinged against the underlying bone. This forms a thrombogenic surface, platelet aggregation, and the formation of a thrombus that is partial, complete, or with secondary embolization. Over time the intimal tear may cause subintimal dissection of the vessel [2].

Pseudoaneurysms are less common and occur as a result of partial transection of the artery. Free rupture is also reported.

The latency period between the injury and the development of cerebrovascular symptoms is a characteristic feature of $\mathrm{BCI}$, and considered an opportunity for initiation of preventive therapy. Approximately $80 \%$ of patients with BCI show no obvious neurologic manifestations at presentation [5]. Studies suggest that 25 to $50 \%$ of patients develop symptoms of BCI as late as 12 hours after the trauma $[6,10-13]$.

\section{Screening}

There seems to be a lack of consensus regarding the optimal diagnostic strategy for detection of $\mathrm{BCI}$, with controversy about the cost-effectiveness of aggressive screening 

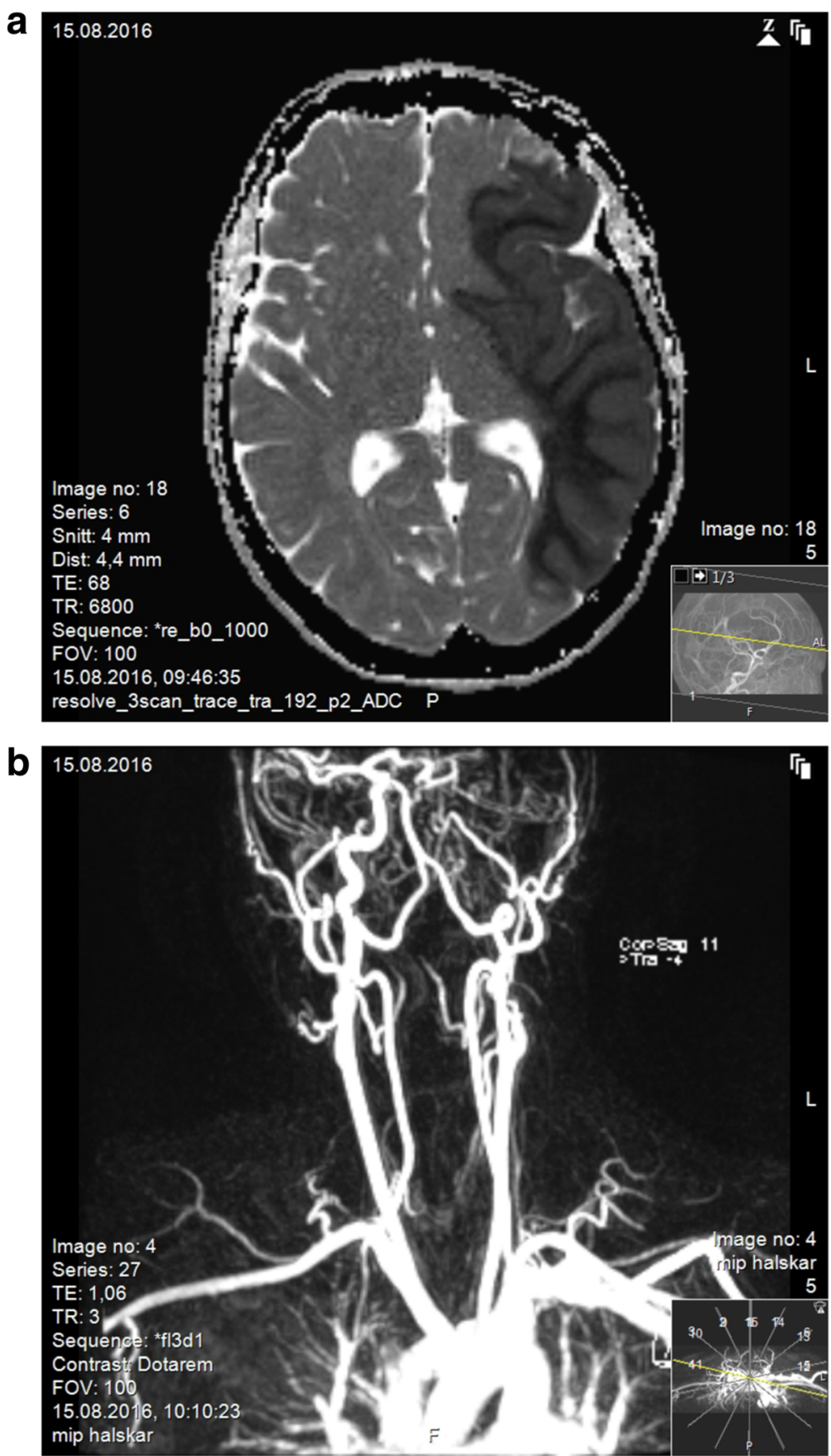

Fig. 2 a Head magnetic resonance imaging taken 17 hours after admittance (top); Shows an extensive left middle cerebral artery infarction. $\mathbf{b}$ Magnetic resonance angiography taken 17 hours after admittance (bottom); Shows an occluded internal carotid artery on the left side approximately $1 \mathrm{~cm}$ above the bifurcation, in addition there is occlusion of the left middle cerebral artery

[14]. With the use of well-compiled screening criteria, patients at risk may be identified early, and preventive treatment initiated.

How to recognize patients at risk? During the years 1990 to 1998 Biffl et al. performed linear regression analysis of a liberally screened population $(n=249)$, and defined four independent risk factors for BCI. In
1996 they initiated a screening of at-risk asymptomatic patients using arteriography based on these criteria [1].

Using this approach, 85 patients (34\%) were diagnosed as having vascular injuries: 65 patients had carotid injuries, 10 had vertebral injuries, and 10 had both carotid and vertebral injuries. Carotid injuries were bilateral in 32 patients. Among 209 asymptomatic patients, cerebrovascular injuries 
Table 1 Characteristic mechanisms of blunt carotidal injury [9]

\begin{tabular}{ll}
\hline Type & Mechanism \\
\hline 1 & $\begin{array}{l}\text { Direct application of force to the neck (seatbelt, strangulation, } \\
\text { near-hanging) }\end{array}$ \\
3 & $\begin{array}{l}\text { Hyperextension and contralateral rotation of the head and neck } \\
\text { Intraoral trauma that affects the internal carotid artery at the } \\
4\end{array}$ \\
\hline
\end{tabular}

were diagnosed in 57 (27\%). This shows a relatively high yield, considering the potentially devastating outcome of these injuries [1].

In 2010 the Eastern Association for the Surgery of Trauma set out to perform a review of all relevant literature concerning management of $\mathrm{BCI}$, and to develop guidelines for screening, diagnosis, and treatment [15].

Screening has clearly increased the number of BCI injuries diagnosed [5-7], and many trauma centers have implemented screening protocols. The optimal screening criteria remain a topic of debate.

In regard of cost-effectiveness, studies have shown screening to be beneficial [14]. The 2016 recommendation of Biffl et al. involves an algorithm based on clinical signs and symptoms that prompt immediate diagnostic evaluation and neurovascular imaging [16].

In the absence of prospective, randomized clinical trials, the current recommendations are based on published observational studies only.

CT angiography is believed to be the most reliable noninvasive screening modality. Sensitivity depends on the number of imaging slices, with 16 slices or more needed for consistent correlation with the results of digital subtraction arteriography (DSA) [17].

DSA remains the gold standard of diagnosis, and is indicated when the level of suspicion is high, despite negative initial imaging results. However, arteriography is an invasive procedure and associated with complications [5]. Another aspect is cost and availability. Arteriography should be reserved for cases where it is required for a definite diagnosis or when an appropriately sensitive CT scanner is not available. MRA has poor specificity (67\%) and sensitivity (50 to $75 \%$ ) compared to DSA and is not recommended [6].

\section{Treatment}

Symptoms, site of injury, severity grade of injury, and associated injuries impact the choice of treatment and follow-up strategy. In the absence of contraindication, such as active hemorrhage, an injury grade-specific recommendation for antithrombotic therapy is given $[18,19]$. Several retrospective studies have reported convincing improvement in neurologic outcome among symptomatic patients, with a reduction in the occurrence of stroke in asymptomatic patients with $\mathrm{BCI}$ receiving antithrombotic therapy compared with those not treated $[5,11,14,18]$.

Heparin is preferred in the acute setting due to its reversibility. There are, however, no randomized trials comparing clinical outcome of different antithrombotic treatment regimens.

For most patients with $\mathrm{BCI}$, inaccessibility of the site of injury precludes direct surgical repair, as the involved vessel is often located at the base of the skull. According to the grading scale for BCI created in 1999 [20], the recommendation is surgical management for patients with accessible Grade II to $\mathrm{V} \mathrm{BCI}$; this in agreement with the guidelines of major trauma societies $[15,21]$.

Follow-up imaging using CT angiography is recommended 7 to 10 days after identification of the cerebrovascular injury, with repeat imaging after 3 months to determine whether long-term antithrombotic therapy is needed.

\section{Conclusions}

$\mathrm{BCI}$, once considered a rare occurrence, has been recognized with increasing frequency in recent years. With the institution of new screening protocols, BCI is now more commonly observed. Early diagnosis and prompt anticoagulation therapy have reduced the occurrence of ischemic neurologic events and disability.

Adequate management requires a high index of suspicion in victims of trauma where the mechanism of injury places the ICA at risk. As illustrated by the case presented here, high-impact trauma resulting in facial fractures represents a risk for $\mathrm{BCI}$. In order to identify these patients at an early stage we emphasize the need for implementation of appropriate screening protocols in the ER [16].

\section{Abbreviations}

BCl: Blunt cerebrovascular injury; CBC: Complete blood count; CT: Computed tomography; DSA: Digital subtraction arteriography; ER: Emergency Room; GCS: Glasgow Coma Scale; ICA: Internal carotid artery; ICU: Intensive Care Unit; MCA: Middle cerebral artery; MRA: Magnetic resonance angiography; MRI: Magnetic resonance imaging

\section{Acknowledgements}

None.

Funding

Not applicable.

Availability of data and materials

Not applicable.

Authors' contributions

All authors contributed in the diagnosis and treatment of the patient and in preparing the final manuscript. All authors read and approved the final manuscript.

Competing interests

The authors declare that they have no competing interests. 


\section{Consent for publication}

Written informed consent for the publication of this case report and any accompanying images was obtained from the patient's husband. A copy of the written consent form is available for review by the Editor-in-Chief of this journal.

\section{Ethics approval and consent to participate}

Not applicable.

\section{Publisher's Note}

Springer Nature remains neutral with regard to jurisdictional claims in published maps and institutional affiliations.

\section{Author details}

'Department of Ear Nose and Throat Surgery, Nordland Hospital, Bodø, Norway. ${ }^{2}$ Department of Oral and Maxillofacial Surgery, Nordland Hospital, Bodø, Norway. ${ }^{3}$ Department of Anesthesiology and Intensive Care, Nordland Hospital, Bodø, Norway. ${ }^{4}$ Institute of Clinical Medicine, University of Tromsø, Tromsø, Norway. ${ }^{5}$ Faculty of Professional Studies, Nord University, Bodø, Norway.

Received: 11 January 2017 Accepted: 9 May 2017

Published online: 02 June 2017

\section{References}

1. Biffl WL, Moore EE, Offner PJ, Brega KE, Franciose RJ, Elliott JP, Burch JM. Optimizing screening for blunt cerebrovascular injuries. Am J Surg. 1999; 178:517-22.

2. Biffl WL, Moore EE, Ryu RK, Offner PJ, Novak Z, Coldwell DM, Franciose RJ, Burch JM. The unrecognized epidemic of blunt carotid arterial injuries: early diagnosis improves neurologic outcome. Ann Surg. 1998;228:462-70.

3. Cogbill TH, Moore EE, Meissner M, Fischer RP, Hoyt DB, Morris JA, Shackford SR, Wallace JR, Ross SE, Ochsner MG. The spectrum of blunt injury to the carotid artery: a multicenter perspective. J Trauma. 1994;37:473-9.

4. Mutze S, Rademacher G, Matthes G, Hosten N, Stengel D. Blunt cerebrovascular injury in patients with blunt multiple trauma: diagnostic accuracy of duplex Doppler US and early CT angiography. Radiology. 2005: 237:884-92.

5. Biffl WL, Ray CE, Moore EE, Franciose RJ, Aly S, Heyrosa MG, Johnson JL, Burch JM, Burch JM. Treatment-related outcomes from blunt cerebrovascular injuries: importance of routine follow-up arteriography. Ann Surg. 2002;235:699-707.

6. Miller PR, Fabian TC, Croce MA, Cagiannos C, Williams JS, Vang M, Qaisi WG, Felker RE, Timmons SD. Prospective screening for blunt cerebrovascular injuries: analysis of diagnostic modalities and outcomes. Ann Surg. 2002;236: 386-95.

7. Bruns BR, Tesoriero R, Kufera J, Sliker C, Laser A, Scalea TM, Stein DM. Blunt cerebrovascular injury screening guidelines: what are we willing to miss? J Trauma Acute Care Surg. 2014;76:691-5

8. van Wessem KJP, Meijer JMR, Leenen LPH, van der Worp HB, Moll FL, de Borst GJ. Blunt traumatic carotid artery dissection still a pitfall? The rationale for aggressive screening. Eur J Trauma Emerg Surg. 2011;37:147-54.

9. Crissey MM, Bernstein EF, Calcaterra TC, Holt GP, DeWeese JA, Rob CG, Satran R, Morris FH, Lipchik EO, ZehI DN, Long JM, Fleming JFR, Petric D, Gurdjian ES, Hardy WG, Thomas LM, Gurdjian ES, Blaise A, Renato WS, Thomas LM, Hare RR, Gaspar MR, Horner TG, Maroon JC, Campbell RL, Hughes JT, Brownell B, Javid H, Ostermiller WE, Heughesh JW, et al. Delayed presentation of carotid intimal tear following blunt craniocervical trauma. Surgery. 1974:75:543-9.

10. Moulakakis KG, Mylonas S, Avgerinos E, Kotsis T, Liapis CD. An update of the role of endovascular repair in blunt carotid artery trauma. Eur J Vasc Endovasc Surg. 2010;40:312-9.

11. Fabian TC, Patton JH, Croce MA, Minard G, Kudsk KA, Pritchard FE. Blunt carotid injury. Importance of early diagnosis and anticoagulant therapy. Ann Surg. 1996;223:513-22.

12. Duke BJ, Ryu RK, Coldwell DM, Brega KE. Treatment of blunt injury to the carotid artery by using endovascular stents: an early experience. J Neurosurg. 1997:87:825-9.

13. Li W, D'Ayala M, Hirshberg A, Briggs W, Wise L, Tortolani A. Comparison of conservative and operative treatment for blunt carotid injuries: analysis of the National Trauma Data Bank. J Vasc Surg. 2010;51:593-9. 599-2.
14. Cothren CC, Moore EE, Ray CE, Ciesla DJ, Johnson JL, Moore JB, Burch JM. Screening for blunt cerebrovascular injuries is cost-effective. Am J Surg. 2005;190:845-9.

15. Bromberg WJ, Collier BC, Diebel LN, Dwyer KM, Holevar MR, Jacobs DG, Kurek SJ, Schreiber MA, Shapiro ML, Vogel TR. Blunt cerebrovascular injury practice management guidelines: the Eastern Association for the Surgery of Trauma. J Trauma. 2010;68:471-7.

16. Biffl WL, Burlew CC, Moore EE. Blunt cerebrovascular injury: Mechanisms, screening, and diagnostic evaluation. UpToDate. 2016. https://www. uptodate.com/contents/blunt-cerebrovascular-injury-mechanisms-screeningand-diagnostic-evaluation. Accessed 16 Mar 2017.

17. Schneidereit NP, Simons R, Nicolaou S, Graeb D, Brown DR, Kirkpatrick A, Redekop G, McKevitt EC, Neyestani A. Utility of screening for blunt vascular neck injuries with computed tomographic angiography. J Trauma. 2006;60: 209-16.

18. Cothren CC, Moore EE, Biffl WL, Ciesla DJ, Ray CE, Johnson JL, Moore JB, Burch JM. Anticoagulation is the gold standard therapy for blunt carotid injuries to reduce stroke rate. Arch Surg. 2004;139:540-6.

19. Biffl WL, Burlew CC, Moore EE. Blunt cerebrovascular injury: Treatment and outcomes. UpToDate. 2016. https://www.uptodate.com/contents/bluntcerebrovascular-injury-treatment-and-outcomes. Accessed 18 Jul 2016.

20. Biffl WL, Moore EE, Offner PJ, Brega KE, Franciose RJ, Burch JM. Blunt carotid arterial injuries: implications of a new grading scale. J Trauma. 1999;47:845-53.

21. Biffl WL, Cothren CC, Moore EE, Kozar R, Cocanour C, Davis JW, Mclntyre RC, West MA, Moore FA. Western Trauma Association critical decisions in trauma: screening for and treatment of blunt cerebrovascular injuries. J Trauma. 2009; 67:1150-3.

\section{Submit your next manuscript to BioMed Central and we will help you at every step:}

- We accept pre-submission inquiries

- Our selector tool helps you to find the most relevant journal

- We provide round the clock customer support

- Convenient online submission

- Thorough peer review

- Inclusion in PubMed and all major indexing services

- Maximum visibility for your research

Submit your manuscript at www.biomedcentral.com/submit
Biomed Central 